(c) 2018 - ISSN 1807-2577

\title{
Acesso a bens e serviços de saúde bucal por escolares de 7 a 10 anos em Vitória-ES
}

\author{
Access to oral health goods and services for school children from 7 to 10 years \\ in Vitória-ES
}

\author{
Maiara Paula Malacarne ZAMBALDI ${ }^{a}$ (D), Maria del Carmem Bisi MOLINA ${ }^{a}$ (D), \\ Camila Bruneli do PRADOa* (D), Edson Theodoro dos SANTOS NETOa (D) \\ aUFES - Universidade Federal do Espírito Santo, Programa de Pós-graduação em Saúde Coletiva, Vitória, ES, Brasil
}

Como citar: Zambaldi MPM, Molina MCB, Prado CB, Santos Neto ET. Acesso a bens e serviços de saúde bucal por escolares de 7 a 10 anos em Vitória-ES. Rev Odontol UNESP. 2021;50:e20210030. https://doi.org/10.1590/1807-2577.03021

\begin{abstract}
Resumo
Introdução: A utilização dos bens e serviços de saúde é considerada uma expressão positiva de acesso, influenciada por diversos fatores. Objetivo: Analisar o acesso aos serviços e bens em saúde bucal, com seus fatores determinantes, entre escolares de 7-10 anos. Material e método: Estudo seccional realizado com escolares de amostra probabilística por conglomerado. Os dados foram coletados através de formulário aplicado às crianças e questionário enviado aos pais/responsáveis. 0 desfecho de interesse foi o acesso aos bens e serviços de saúde odontológicos, a partir das variáveis "primeira consulta odontológica" e "posse de escova dental própria". As variáveis independentes (características infantis, maternas e socioeconômicas) que apresentaram significância estatística $\leq 0,10$, na análise bivariada, foram incluídas no modelo de Regressão Logística Multivariada. 0 nível de significância adotado no modelo final foi de 5\%. Resultado: Associaram à posse de escova dental: idades de 8 (OR=3,303; IC95\%=1,135 - 9,610), 9 (OR=3,452; IC95\%=1,176 - 10,137) e 10 anos (OR=6,901; IC95\% = 1,468 - 32,434); tabagismo materno (OR=4,509; IC95\%= 2,021-10,060). Associados à primeira consulta odontológica: idades de 9 (OR=2,321; IC $95 \%=1,340-4,022)$ e 10 anos (OR=3,155; IC95\% = 1,687 - 5,899); escola privada (OR=3,691; IC95\% =1,343-10,145); dois ou mais quartos na residência (OR: 3,691; IC95\% = 1,343 - 10,145) e posse de escova dental própria (OR=2,065; IC95\% = 1,009 - 4,226). Conclusão: Os resultados apontam para desigualdade no acesso à saúde odontológica. Crianças mais velhas, com mães não fumantes e melhores condições socioeconômicas apresentam maior acesso aos serviços e bens em saúde bucal.
\end{abstract}

Descritores: Acesso aos serviços de saúde; serviços de saúde bucal; saúde bucal; criança.

\begin{abstract}
Introduction: The use of health goods and services is considered a positive expression of access, influenced by several factors. Objective: Analyze access to oral health services and goods, and determining factors, among schoolchildren aged 7-10 years. Material and method: Cross-sectional study carried out with students from a probabilistic cluster sample. Data were collected through a form applied to children and a questionnaire sent to parents/guardians. The outcome of interest was access to dental health goods and services, based on the variables "first dental appointment" and "own a toothbrush". Independent variables (child, maternal and socioeconomic characteristics) that presented statistical significance $\leq 0.10$ in the bivariate analysis were included in the Multivariate Logistic Regression model. The significance level adopted in the final model was 5\%. Result: They associated with having a toothbrush: ages of $8(\mathrm{OR}=3.303 ; 95 \% \mathrm{CI}=1.135-9.610), 9(\mathrm{OR}=3.452$; $95 \% \mathrm{CI}=1.176-10.137)$ and 10 years $(\mathrm{OR}=6.901 ; 95 \% \mathrm{CI}=1.468-32.434)$; maternal smoking $(\mathrm{OR}=4.509 ; 95 \% \mathrm{CI}=2.021-10.060)$. Associated with the first dental appointment: ages $9(\mathrm{OR}=2.321$; $95 \% \mathrm{CI}=1.340-4.022)$ and 10 years $(\mathrm{OR}=3.155 ; 95 \% \mathrm{CI}=1.687-5.899)$; private school $(\mathrm{OR}=3.691$; $95 \% \mathrm{CI}=1.343-10.145$ ); two or more bedrooms in the residence (OR: $3.691 ; 95 \% \mathrm{CI}=1.343-10.145$ ) and own a toothbrush $(\mathrm{OR}=2.065 ; 95 \% \mathrm{CI}=1.009-4.226)$. Conclusion: The results point to inequality in access
\end{abstract}


to dental health. Older children, with uneducated mothers and better socioeconomic conditions have greater access to services and goods in oral health.

Descriptors: Health services accessibility; dental health services; oral health; child.

\section{INTRODUÇÃO}

No Brasil, o princípio de equidade no acesso aos serviços de saúde norteia o sistema de saúde brasileiro, de maneira que o Sistema Único de Saúde (SUS) tem como objetivo garantir acesso 'universal, igualitário e gratuito' aos serviços de saúde ${ }^{1}$. Contudo, a determinação do conceito e da terminologia do acesso é temática complexa, de modo que parece não haver um consenso a respeito dos múltiplos significados dados ao termo ${ }^{2,3}$.

Para Donabedian ${ }^{4}$, a acessibilidade estaria relacionada às características do sistema de saúde que facilitariam ou obstruiriam a utilização dos serviços pelos usuários em potencial. Penchansky, Thomas $^{2}$ utilizaram o termo acesso para definir a relação de ajuste entre as características do sistema de saúde e as características e expectativas dos usuários.

Andersen e Newman ${ }^{5}$ abordaram que o conceito de acesso estaria ligado à entrada inicial do indivíduo ao sistema de saúde e influenciaria a utilização dos serviços de saúde por meio dos fatores capacitantes, predisponentes e necessidades em saúde. Os fatores predisponentes seriam aqueles relacionados às características demográficas, sociais e às crenças dos indivíduos. Os fatores capacitantes considerariam as características familiares e da comunidade em que o sujeito estaria inserido. Já as necessidades de saúde representariam o estado de saúde do indivíduo, seja ele percebido ou diagnosticado.

Em análises posteriores do modelo, Andersen ${ }^{6}$ incorpora novas dimensões de acesso denominadas de: acesso potencial; acesso realizado, referido como a utilização dos serviços de saúde propriamente dita; e acesso eficiente.

0 conceito de acesso é muito complexo e varia conforme a abordagem dos autores. De maneira similar, sua relação com a utilização dos serviços de saúde também é pouco clara 3 .

Entretanto, a utilização dos serviços de saúde pode ser analisada como um indicador de acesso $^{7}$, ao considerarmos que no decorrer da vida a maioria dos indivíduos depara-se com situações de necessidade de cuidado por algum profissional de saúde.

A utilização dos serviços de saúde representa o centro do funcionamento dos sistemas de saúde ${ }^{3}$, e diversos fatores são capazes de influenciar sua utilização. Assim, de maneira geral, o acesso tende a refletir as desigualdades sociais existentes entre os grupos populacionais ${ }^{8}$.

As desigualdades de acesso podem ocorrer nos diversos tipos de serviços de saúde, inclusive nos odontológicos, de modo que fatores relacionados à renda, escolaridade, idade, sexo, necessidades em saúde, entre outros, influenciam na obtenção ou não do cuidado em saúde bucal. Em relação às características socioeconômicas, observa-se que a utilização dos serviços é desigual e a favor dos mais ricos ${ }^{9}$, e que o consumo tende a aumentar à medida que a renda e a escolaridade aumentam ${ }^{7,10}$. Quanto às necessidades de saúde, a doença é protagonista na procura de serviços de saúde ${ }^{9}$, pois indivíduos com dor dentária, diagnóstico de doenças crônicas e autopercepção de saúde como ruim, possuem maiores frequências de acesso aos serviços de saúde odontológicos 7,9 .

Essas desigualdades observadas no acesso aos serviços de saúde bucal podem ocorrer tanto entre as regiões brasileiras como dentro de um mesmo estado ou município. A cidade de Vitória, capital do Estado do Espírito Santo, Brasil, destaca-se por apresentar uma organizada rede de saúde bucal ${ }^{11}$.

Todavia, as características de desenvolvimento favorável podem não refletir na utilização igualitária pela população aos serviços de saúde, pois a equidade na utilização dos serviços depende, além de bons indicadores, da disponibilidade de oferta dos serviços de saúde, dos recursos humanos e das tecnologias em saúde disponíveis na região ${ }^{8}$. Portanto, o objetivo deste 
estudo foi analisar o acesso aos serviços e bens em saúde bucal, com seus fatores determinantes, entre escolares de 7-10 anos no município de Vitória-ES.

\section{METODOLOGIA}

Este estudo é parte de um estudo denominado "Nutrição e Saúde dos escolares de 7 a 10 anos matriculados em escolas públicas e privadas de Vitória-ES" (Estudo SAUDES). 0 estudo é de delineamento seccional com amostragem probabilística.

Para estimar a amostra do estudo foi utilizado o Censo Brasileiro de 2002 para identificar a população total entre 7 e 10 anos de idade no município de Vitória. 0 relatório de matrículas, a partir de 2006, do Ministério da Educação, foi utilizado para determinar a proporção de crianças matriculadas em escolas públicas e privadas. Assim, a amostra foi estratificada em três etapas. A escola foi a primeira etapa, a sala de aula a segunda e o sorteio das crianças a terceira etapa. A amostra final contou com crianças oriundas de 29 escolas públicas e 06 privadas. Os dados foram estratificados por cotas de tipo de escola (pública e particular), sexo (masculino e feminino) e por idade (7, 8, 9 e 10 anos). Foi definido um número de 40 crianças por escola ${ }^{12}$. Assim, a amostra final consistiu de 1282 escolares, com proporções representativas de sexo, idade e rede de ensino.

A coleta dos dados foi realizada por meio de dois instrumentos. 0 primeiro consistia em um formulário aplicado às crianças por pesquisadores treinados, no qual eram abordadas questões relacionadas às práticas de alimentação e de saúde bucal. 0 segundo instrumento tratava-se de um questionário para autopreenchimento pelas mães/cuidadoras das crianças com questões relativas aos aspectos socioeconômicos, alimentação e atividade física. 0 presente estudo investigou o acesso aos bens e serviços de saúde odontológicos por meio da variável de utilização $2,5,6$. E de consulta odontológica, pelo menos uma vez na vida ("primeira consulta odontológica") e por meio da variável "posse de escova dental própria", sem compartilhamento domiciliar, associadas com as variáveis independentes relacionadas às características infantis, maternas e socioeconômicas. Para tanto, o conceito de acesso adotado foi conforme o descrito por Andersen ${ }^{6}$, em que a dimensão de acesso realizado é considerada como a utilização dos serviços de saúde propriamente dita.

As características infantis testadas foram: experiência de cárie, sexo, idade, tipo de escola, raça/cor, doença respiratória, companhia no almoço, companhia no jantar e prematuridade. As características maternas testadas foram: idade, escolaridade, tabagismo e ocupação. De maneira similar, as características socioeconômicas testadas foram: residência conjunta pai e mãe, aglomeração humana domiciliar, tipo de casa, acesso a água tratada, acesso a rede de esgoto, acesso a água filtrada, quantidade de quartos no domicílio, escolaridade do chefe da residência e classe socioeconômica.

Com o auxílio do programa Statistical Package for the Social Sciences for Windows 17.0 (SPSS), investigou-se a existência de associação entre as variáveis referentes à "posse de escova dental própria" e "primeira consulta odontológica" determinantes do acesso aos serviços de saúde e às variáveis independentes previamente agrupadas em características infantis, maternas e socioeconômicas. As associações bivariadas foram testadas por meio do teste o Qui-quadrado com associação de Yates.

As variáveis independentes que apresentaram significância menor ou igual a $0,10 \mathrm{com}$ o desfecho, quando a análise bivariada foi conduzida, foram incluídas no modelo de Regressão Logística Multivariada, segundo método Enter. 0 nível de significância adotado para as variáveis no modelo final foi $5 \%$, e para cada variável independente foram obtidos as odds ratio e os intervalos de confiança.

As variáveis relacionadas à escolaridade do chefe e à escolaridade materna não foram incluídas no modelo de análise final, por estarem fortemente associadas entre si e com a classe socioeconômica ( $\mathrm{p}$-valor $<5 \%$ ). Porém, a classe socioeconômica funcionou como variável de ajuste. 
A pesquisa principal do qual o presente estudo se originou foi aprovada pelo Comitê de Ética em Pesquisa do Centro Biomédico da Universidade Federal do Espírito Santo em 26/10/06, sob número de registro no CEP/UFES 089/06. A realização do estudo nas escolas públicas foi autorizada oficialmente pela Secretaria Municipal de Educação do Município de Vitória, e todas as crianças participantes da pesquisa possuíam o Termo de Consentimento Livre e Esclarecido assinado pelos responsáveis. Também foi obtida autorização formal do coordenador do estudo para realização desta pesquisa.

\section{RESULTADO}

A Tabela 1 mostra os resultados da análise bivariada referente às características infantis e às variáveis desfecho: "posse de escova dental própria" e "primeira consulta odontológica". As características infantis que se relacionaram com a variável dependente "posse de escova dental própria" foram: experiência de cárie $(\mathrm{p}=0,005)$, idade $(\mathrm{p}=0,001)$, tipo de escola $(\mathrm{p}=0,060)$ e raça/cor $(\mathrm{p}<0,001)$. Quando o desfecho referente à "primeira consulta odontológica" foi considerado, as mesmas variáveis permaneceram associadas, de maneira que o nível descritivo do teste foi: experiência de cárie $(\mathrm{p}<0,001)$, idade $(\mathrm{p}<0,001)$, tipo de escola $(\mathrm{p}<0,001)$ e raça/cor $(\mathrm{p}<0,001)$.

As características maternas e socioeconômicas estão demonstradas na Tabela 2. Dentre as características maternas que se relacionaram com os desfechos estão a: escolaridade materna, tanto com posse de escova dental própria $(\mathrm{p}=0,007)$ quanto com primeira consulta odontológica $(\mathrm{p}<0,001)$, e tabagismo materno, tanto com posse de escova dental própria $(\mathrm{p}=0,011)$ quanto primeira consulta odontológica $(\mathrm{p}=0,100)$.

Quanto às características socioeconômicas, a residência conjunta com pai e mãe $(p=0,029)$ e a quantidade de quartos $(\mathrm{p}=0,001)$ associaram-se apenas com a primeira consulta odontológica, e a aglomeração humana domiciliar apenas com a posse de escova dental própria $(\mathrm{p}<0,001)$. As outras variáveis que se relacionaram com ambas as variáveis dependentes foram: acesso a água filtrada; escolaridade do chefe e classe socioeconômica. Além disso, essas variáveis de desfecho estão também associadas entre si $(\mathrm{p}=0,038)$.

No modelo de Regressão Logística Multivariada, permaneceram associadas ao desfecho referente à posse de escova dental própria as variáveis: idade (8 anos, 9 anos e 10 anos); tabagismo materno e primeira consulta odontológica. Quanto à primeira consulta odontológica, as variáveis independentes associadas foram idade ( 9 anos e 10 anos); tipo de escola; quantidade de quartos e posse de escova dental própria. Os resultados das análises multivariadas estão descritos nas Tabelas 3 e 4 .

Tabela 1. Análise bivariada das características infantis com os desfechos de primeira consulta odontológica e posse de escova dental própria da amostra de 1282 crianças do Estudo SAÚDES. Vitória- ES, 2007

\begin{tabular}{|c|c|c|c|c|c|c|c|c|c|}
\hline \multirow[t]{2}{*}{ Variáveis } & \multirow[t]{2}{*}{ Categorias } & \multicolumn{2}{|c|}{$\begin{array}{l}\text { Escova dental } \\
\text { própria }\end{array}$} & \multirow[t]{2}{*}{ Total } & \multirow{2}{*}{$\begin{array}{c}\mathbf{P} \\
\text { valor* }\end{array}$} & \multicolumn{2}{|c|}{$\begin{array}{c}\text { Primeira consulta } \\
\text { odontológica }\end{array}$} & \multirow[t]{2}{*}{ Total } & \multirow[t]{2}{*}{ P-valor* } \\
\hline & & Sim & Não & & & Sim & Não & & \\
\hline \multirow{2}{*}{$\begin{array}{c}\text { Experiência de } \\
\text { Cárie }\end{array}$} & Sim & $\begin{array}{c}560 \\
(98,9 \%)\end{array}$ & $6(1,1 \%)$ & 566 & 0,005 & $\begin{array}{c}501 \\
(89,1 \%)\end{array}$ & $61(10,9 \%)$ & 562 & 0,000 \\
\hline & Não & $\begin{array}{c}489 \\
(96,1 \%)\end{array}$ & $\begin{array}{c}20 \\
(3,9 \%)\end{array}$ & 509 & & $\begin{array}{c}411 \\
(81,2 \%)\end{array}$ & $95(18,8 \%)$ & 506 & \\
\hline \multirow{2}{*}{ Sexo da Criança } & Masculino & $524(97,4 \%)$ & $14(2,6 \%)$ & 538 & 0,486 & $\begin{array}{c}460 \\
(85,7 \%)\end{array}$ & $77(14,3 \%)$ & 537 & 0,955 \\
\hline & Feminino & $\begin{array}{c}729 \\
(98,0 \%)\end{array}$ & $\begin{array}{c}15 \\
(2,0 \%)\end{array}$ & 744 & & $\begin{array}{c}633 \\
(85,8 \%)\end{array}$ & $\begin{array}{c}105 \\
(14,2 \%)\end{array}$ & 738 & \\
\hline
\end{tabular}


Tabela 1. Continuação...

\begin{tabular}{|c|c|c|c|c|c|c|c|c|c|}
\hline \multirow[t]{2}{*}{ Variáveis } & \multirow[t]{2}{*}{ Categorias } & \multicolumn{2}{|c|}{$\begin{array}{c}\text { Escova dental } \\
\text { própria }\end{array}$} & \multirow[t]{2}{*}{ Total } & \multirow{2}{*}{$\begin{array}{c}\mathbf{P} \\
\text { valor* }\end{array}$} & \multicolumn{2}{|c|}{$\begin{array}{c}\text { Primeira consulta } \\
\text { odontológica }\end{array}$} & \multirow[t]{2}{*}{ Total } & \multirow[t]{2}{*}{ P-valor* } \\
\hline & & Sim & Não & & & Sim & Não & & \\
\hline \multirow{4}{*}{$\begin{array}{l}\text { Idade da } \\
\text { Criança }\end{array}$} & Sete & $\begin{array}{c}238 \\
(94,4 \%)\end{array}$ & $\begin{array}{c}14 \\
(5,6 \%)\end{array}$ & 252 & 0,001 & $\begin{array}{c}196 \\
(77,8 \%)\end{array}$ & $56(22,2 \%)$ & 252 & 0,000 \\
\hline & Oito & $\begin{array}{c}352 \\
(98,1 \%)\end{array}$ & $7(1,9 \%)$ & 359 & & $\begin{array}{c}296 \\
(82,7 \%)\end{array}$ & $62(17,3 \%)$ & 358 & \\
\hline & Nove & $\begin{array}{c}354 \\
(98,3 \%)\end{array}$ & $6(1,7 \%)$ & 360 & & $\begin{array}{c}317 \\
(89,3 \%)\end{array}$ & $38(10,7 \%)$ & 355 & \\
\hline & Dez & $\begin{array}{c}309 \\
(99,3 \%)\end{array}$ & $2(0,7 \%)$ & 311 & & $\begin{array}{c}284 \\
(91,6 \%)\end{array}$ & $26(8,4 \%)$ & 310 & \\
\hline \multirow{2}{*}{ Tipo de Escola } & Pública & $\begin{array}{c}1032 \\
(97,3 \%)\end{array}$ & $\begin{array}{c}29 \\
(2,7 \%)\end{array}$ & 1061 & 0,060 & $\begin{array}{c}878 \\
(83,3 \%)\end{array}$ & $\begin{array}{c}176 \\
(16,7 \%)\end{array}$ & 1054 & 0,000 \\
\hline & Privada & $\begin{array}{c}221 \\
(100 \%)\end{array}$ & $0(0 \%)$ & 221 & & $\begin{array}{c}215 \\
(97,3 \%)\end{array}$ & $6(2,7 \%)$ & 221 & \\
\hline \multirow{3}{*}{$\begin{array}{c}\text { Cor/Raça da } \\
\text { Criança }\end{array}$} & Branco & $\begin{array}{c}417 \\
(99,5 \%)\end{array}$ & $2(0,5 \%)$ & 419 & 0,000 & $\begin{array}{c}380 \\
(91,3 \%)\end{array}$ & $36(8,7 \%)$ & 416 & 0,000 \\
\hline & Preto & $\begin{array}{c}157 \\
(93,4 \%)\end{array}$ & $\begin{array}{c}11 \\
(6,6 \%)\end{array}$ & 168 & & $\begin{array}{c}135 \\
(80,8 \%)\end{array}$ & $32(19,2 \%)$ & 167 & \\
\hline & Pardo & $\begin{array}{c}638 \\
(97,8 \%)\end{array}$ & $\begin{array}{c}14 \\
(2,2 \%)\end{array}$ & 652 & & $\begin{array}{c}540 \\
(83,1 \%)\end{array}$ & $\begin{array}{c}110 \\
(16,9 \%)\end{array}$ & 650 & \\
\hline \multirow{2}{*}{$\begin{array}{c}\text { Doença } \\
\text { Respiratória }\end{array}$} & Sim & 191 (99\%) & $2(1 \%)$ & 193 & 0,295 & $\begin{array}{c}167 \\
(87,0 \%)\end{array}$ & $25(13,0 \%)$ & 192 & 0,544 \\
\hline & Não & $\begin{array}{c}1021 \\
(97,5 \%)\end{array}$ & $\begin{array}{c}26 \\
(2,5 \%)\end{array}$ & 1047 & & $\begin{array}{c}888 \\
(85,3 \%)\end{array}$ & $\begin{array}{c}153 \\
(14,7 \%)\end{array}$ & 1041 & \\
\hline \multirow{3}{*}{$\begin{array}{c}\text { Companhia no } \\
\text { Almoço }\end{array}$} & Sozinha & $\begin{array}{c}170 \\
(98,3 \%)\end{array}$ & $3(1,7 \%)$ & 173 & 0,752 & $\begin{array}{c}139 \\
(80,8 \%)\end{array}$ & $33(19,2 \%)$ & 172 & 0,135 \\
\hline & $\begin{array}{l}\text { Com alguém da } \\
\text { família }\end{array}$ & $\begin{array}{c}910 \\
(97,5 \%)\end{array}$ & $\begin{array}{c}23 \\
(2,5 \%)\end{array}$ & 933 & & $\begin{array}{c}803 \\
(86,6 \%)\end{array}$ & $\begin{array}{c}124 \\
(13,4 \%)\end{array}$ & 927 & \\
\hline & Outras pessoas & $\begin{array}{c}166 \\
(98,2 \%)\end{array}$ & $3(1,8 \%)$ & 169 & & $\begin{array}{c}145 \\
(85,8 \%)\end{array}$ & $24(14,2 \%)$ & 169 & \\
\hline \multirow{3}{*}{$\begin{array}{c}\text { Companhia no } \\
\text { Jantar }\end{array}$} & Sozinha & $\begin{array}{c}126 \\
(97,7 \%)\end{array}$ & $3(2,3 \%)$ & 129 & 0,528 & $\begin{array}{c}103 \\
(79,8 \%)\end{array}$ & $26(20,2 \%)$ & 129 & 0,107 \\
\hline & $\begin{array}{l}\text { Com alguém da } \\
\text { família }\end{array}$ & $\begin{array}{c}1011 \\
(97,9 \%)\end{array}$ & $\begin{array}{c}22 \\
(2,1 \%)\end{array}$ & 1033 & & $\begin{array}{c}885 \\
(86,3 \%)\end{array}$ & $\begin{array}{c}141 \\
(13,7 \%)\end{array}$ & 1026 & \\
\hline & Outras pessoas & $99(96,1 \%)$ & $4(3,9 \%)$ & 103 & & $91(88,3 \%)$ & $12(11,6 \%)$ & 103 & \\
\hline \multirow{2}{*}{ Prematuro } & Sim & $\begin{array}{c}119 \\
(98,3 \%)\end{array}$ & $2(1,7 \%)$ & 121 & 1,000 & $\begin{array}{c}104 \\
(86,7 \%)\end{array}$ & $16(13,3 \%)$ & 120 & 0,815 \\
\hline & Não & $\begin{array}{c}1079 \\
(97,7 \%) \\
\end{array}$ & $\begin{array}{r}25 \\
(2,3 \%) \\
\end{array}$ & 1104 & & $\begin{array}{c}943 \\
(85,9 \%) \\
\end{array}$ & $\begin{array}{c}155 \\
(14,1 \%) \\
\end{array}$ & 1098 & \\
\hline
\end{tabular}

*Teste qui-quadrado

Tabela 2. Análise bivariada das características maternas e socioeconômicas com os desfechos de primeira consulta odontológica e posse de escova dental própria da amostra de 1282 crianças do Estudo SAÚDES. Vitória- ES, 2007

\begin{tabular}{|c|c|c|c|c|c|c|c|c|c|}
\hline \multirow{2}{*}{ Variáveis } & \multirow{2}{*}{ Categorias } & \multicolumn{2}{|c|}{$\begin{array}{l}\text { Escova dental } \\
\text { própria }\end{array}$} & \multirow{2}{*}{ Total } & \multirow{2}{*}{$\begin{array}{c}\mathbf{P} \\
\text { valor* }\end{array}$} & \multicolumn{2}{|c|}{$\begin{array}{c}\text { Primeira consulta } \\
\text { odontológica }\end{array}$} & \multirow{2}{*}{ Total } & \multirow{2}{*}{$\begin{array}{c}\mathbf{P} \\
\text { valor* }\end{array}$} \\
\hline & & Sim & Não & & & Sim & Não & & \\
\hline \multirow{2}{*}{ Idade materna } & Até 34 anos & $\begin{array}{c}1034 \\
(97,8 \%)\end{array}$ & $\begin{array}{c}23 \\
(2,2 \%)\end{array}$ & 1057 & 0,653 & $\begin{array}{c}898 \\
(85,4 \%)\end{array}$ & $153(14,6 \%)$ & 1051 & \multirow[t]{2}{*}{0,531} \\
\hline & 35 ou mais & $\begin{array}{c}219 \\
(97,3 \%)\end{array}$ & $6(2,7 \%)$ & 225 & & $\begin{array}{c}195 \\
(87,1 \%)\end{array}$ & $29(12,9 \%)$ & 224 & \\
\hline
\end{tabular}


Tabela 2. Continuação...

\begin{tabular}{|c|c|c|c|c|c|c|c|c|c|}
\hline \multirow{2}{*}{ Variáveis } & \multirow{2}{*}{ Categorias } & \multicolumn{2}{|c|}{$\begin{array}{c}\text { Escova dental } \\
\text { própria }\end{array}$} & \multirow{2}{*}{ Total } & \multirow{2}{*}{$\begin{array}{c}\mathbf{P} \\
\text { valor* }\end{array}$} & \multicolumn{2}{|c|}{$\begin{array}{c}\text { Primeira consulta } \\
\text { odontológica }\end{array}$} & \multirow{2}{*}{ Total } & \multirow{2}{*}{$\begin{array}{c}\mathbf{P} \\
\text { valor }\end{array}$} \\
\hline & & Sim & Não & & & Sim & Não & & \\
\hline \multirow{4}{*}{$\begin{array}{c}\text { Escolaridade } \\
\text { materna }\end{array}$} & 1 a 4 anos & $\begin{array}{c}127 \\
(96,2 \%)\end{array}$ & $5(3,8 \%)$ & 132 & 0,007 & $\begin{array}{c}108 \\
(83,1 \%)\end{array}$ & $22(16,9 \%)$ & 130 & \multirow[t]{4}{*}{0,000} \\
\hline & 5 a 8 anos & $\begin{array}{c}346 \\
(96,1 \%)\end{array}$ & $\begin{array}{c}14 \\
(3,9 \%)\end{array}$ & 360 & & $\begin{array}{c}284 \\
(79,8 \%)\end{array}$ & $72(20,2 \%)$ & 356 & \\
\hline & 9 a 11 anos & $\begin{array}{c}509 \\
(98,3 \%)\end{array}$ & $9(1,7 \%)$ & 518 & & $\begin{array}{c}442 \\
(85,5 \%)\end{array}$ & $75(14,5 \%)$ & 517 & \\
\hline & 12 anos ou mais & $\begin{array}{c}245 \\
(100 \%)\end{array}$ & $0(0 \%)$ & 245 & & $\begin{array}{c}235 \\
(95,9 \%)\end{array}$ & $10(4,1 \%)$ & 245 & \\
\hline \multirow{2}{*}{$\begin{array}{c}\text { Tabagismo } \\
\text { materno }\end{array}$} & Sim & $\begin{array}{c}149 \\
(94,9 \%)\end{array}$ & $8(5,1 \%)$ & 157 & 0,011 & $\begin{array}{c}127 \\
(81,4 \%)\end{array}$ & $29(18,6 \%)$ & 156 & \multirow[t]{2}{*}{0,100} \\
\hline & Não & $\begin{array}{c}1104 \\
(98,1 \%)\end{array}$ & $\begin{array}{c}21 \\
(1,9 \%)\end{array}$ & 1125 & & $\begin{array}{c}966 \\
(86,3 \%)\end{array}$ & $153(13,7 \%)$ & 1119 & \\
\hline \multirow{3}{*}{$\begin{array}{c}\text { Ocupação } \\
\text { materna }\end{array}$} & $\begin{array}{c}\text { Empregada/ } \\
\text { Profissional } \\
\text { liberal }\end{array}$ & $\begin{array}{c}763 \\
(97,6 \%)\end{array}$ & $\begin{array}{c}19 \\
(2,4 \%)\end{array}$ & 782 & 0,753 & $\begin{array}{c}677 \\
(87,1 \%)\end{array}$ & $100(12,9 \%)$ & 777 & \multirow[t]{3}{*}{0,286} \\
\hline & Desempregadas & $\begin{array}{c}226 \\
(97,4 \%)\end{array}$ & $6(2,6 \%)$ & 232 & & $\begin{array}{c}192 \\
(83,1 \%)\end{array}$ & $39(16,7 \%)$ & 231 & \\
\hline & Dona de casa & $\begin{array}{c}236 \\
(98,3 \%)\end{array}$ & $4(1,7 \%)$ & 240 & & $\begin{array}{c}204 \\
(85,3 \%)\end{array}$ & $35(14,7 \%)$ & 239 & \\
\hline \multirow{2}{*}{$\begin{array}{c}\text { Residência } \\
\text { conjunta pai e } \\
\text { mãe }\end{array}$} & Sim & $\begin{array}{c}326 \\
(96,7 \%)\end{array}$ & $\begin{array}{c}11 \\
(3,3 \%)\end{array}$ & 337 & 0,179 & $\begin{array}{c}275 \\
(82,1 \%)\end{array}$ & $60(17,9 \%)$ & 335 & \multirow[t]{2}{*}{0,029} \\
\hline & Não & $\begin{array}{c}894 \\
(98,0 \%)\end{array}$ & $\begin{array}{c}18 \\
(2,0 \%)\end{array}$ & 912 & & $\begin{array}{c}789 \\
(87,0 \%)\end{array}$ & $118(13 \%)$ & 907 & \\
\hline \multirow{2}{*}{$\begin{array}{c}\text { Aglomeração } \\
\text { humana } \\
\text { domiciliar }\end{array}$} & Até 5 pessoas & $\begin{array}{c}985 \\
(98,5 \%)\end{array}$ & $\begin{array}{c}15 \\
(1,5 \%)\end{array}$ & 1000 & 0,000 & $\begin{array}{c}862 \\
(86,6 \%)\end{array}$ & $133(13,4 \%)$ & 995 & \multirow[t]{2}{*}{0,147} \\
\hline & $\begin{array}{c}\text { Mais de } 5 \\
\text { pessoas }\end{array}$ & $\begin{array}{c}224 \\
(94,5 \%)\end{array}$ & $\begin{array}{c}13 \\
(5,5 \%)\end{array}$ & 237 & & $\begin{array}{c}195 \\
(83,0 \%)\end{array}$ & $40(17,0 \%)$ & 235 & \\
\hline \multirow{3}{*}{ Tipo de casa } & Própria & $\begin{array}{c}880 \\
(97,9 \%)\end{array}$ & $\begin{array}{c}19 \\
(2,1 \%)\end{array}$ & 899 & 0,214 & $\begin{array}{c}776 \\
(86,8 \%)\end{array}$ & $118(13,2 \%)$ & 894 & \multirow[t]{3}{*}{0,140} \\
\hline & Cedida & $\begin{array}{c}236 \\
(96,3 \%)\end{array}$ & $9(3,7 \%)$ & 245 & & $\begin{array}{c}199 \\
(81,9 \%)\end{array}$ & $44(18,1 \%)$ & 243 & \\
\hline & Alugada & $\begin{array}{c}107 \\
(99,1 \%)\end{array}$ & $1(0,9 \%)$ & 108 & & $\begin{array}{c}94 \\
(87,0 \%)\end{array}$ & $14(13,0 \%)$ & 108 & \\
\hline \multirow{2}{*}{$\begin{array}{c}\text { Acesso a água } \\
\text { tratada }\end{array}$} & Sim & $\begin{array}{c}1232 \\
(97,7 \%)\end{array}$ & $\begin{array}{c}29 \\
(2,3 \%)\end{array}$ & 1261 & 1,000 & $\begin{array}{c}1076 \\
(85,8 \%)\end{array}$ & $178(14,2 \%)$ & 1254 & \multirow[t]{2}{*}{0,513} \\
\hline & Não & $20(100 \%)$ & $0(0 \%)$ & 20 & & $\begin{array}{c}16 \\
(80,0 \%)\end{array}$ & $4(20,0 \%)$ & 20 & \\
\hline \multirow{2}{*}{$\begin{array}{l}\text { Acesso a rede } \\
\text { de esgoto }\end{array}$} & Sim & $\begin{array}{c}1191 \\
(97,7 \%)\end{array}$ & $\begin{array}{c}28 \\
(2,3 \%)\end{array}$ & 1219 & 0,543 & $\begin{array}{c}1042 \\
(85,9 \%)\end{array}$ & $171(14,1 \%)$ & 1213 & \multirow[t]{2}{*}{0,807} \\
\hline & Não & $\begin{array}{c}32 \\
(97,0 \%)\end{array}$ & $1(3,0 \%)$ & 33 & & $\begin{array}{c}27 \\
(84,4 \%)\end{array}$ & $5(15,6 \%)$ & 32 & \\
\hline \multirow{2}{*}{$\begin{array}{l}\text { Acesso a água } \\
\text { filtrada }\end{array}$} & Sim & $\begin{array}{c}1060 \\
(98,3 \%)\end{array}$ & $\begin{array}{c}18 \\
(1,7 \%)\end{array}$ & 1078 & 0,000 & $\begin{array}{c}931 \\
(86,8 \%)\end{array}$ & $141(13,2 \%)$ & 1072 & \multirow[t]{2}{*}{0,006} \\
\hline & Não & $\begin{array}{c}139 \\
(92,7 \%)\end{array}$ & $\begin{array}{c}11 \\
(7,3 \%)\end{array}$ & 150 & & $\begin{array}{c}117 \\
(78,5 \%)\end{array}$ & $32(21,5 \%)$ & 149 & \\
\hline \multirow{2}{*}{$\begin{array}{l}\text { Quantidade de } \\
\text { quartos }\end{array}$} & Um & $\begin{array}{c}154 \\
(96,8 \%)\end{array}$ & $5(3,2 \%)$ & 159 & 0,454 & $\begin{array}{c}123 \\
(77,3 \%)\end{array}$ & $36(22,7 \%)$ & 159 & \multirow[t]{2}{*}{0,001} \\
\hline & Dois ou mais & $\begin{array}{c}1072 \\
(97,8 \%)\end{array}$ & $\begin{array}{c}24 \\
(2,2 \%)\end{array}$ & 1096 & & $\begin{array}{c}946 \\
(86,9 \%)\end{array}$ & $143(13,1 \%)$ & 1089 & \\
\hline
\end{tabular}


Tabela 2. Continuação...

\begin{tabular}{|c|c|c|c|c|c|c|c|c|c|}
\hline \multirow{2}{*}{ Variáveis } & \multirow{2}{*}{ Categorias } & \multicolumn{2}{|c|}{$\begin{array}{c}\text { Escova dental } \\
\text { própria }\end{array}$} & \multirow{2}{*}{ Total } & \multirow{2}{*}{$\begin{array}{c}\mathbf{P} \\
\text { valor* }\end{array}$} & \multicolumn{2}{|c|}{$\begin{array}{l}\text { Primeira consulta } \\
\text { odontológica }\end{array}$} & \multirow{2}{*}{ Total } & \multirow{2}{*}{$\begin{array}{c}\mathbf{P} \\
\text { valor* }\end{array}$} \\
\hline & & Sim & Não & & & Sim & Não & & \\
\hline \multirow{4}{*}{$\begin{array}{l}\text { Escolaridade } \\
\text { do chefe }\end{array}$} & 1 a 4 anos & $\begin{array}{c}157 \\
(96,3 \%)\end{array}$ & $6(3,7 \%)$ & 163 & 0,001 & $\begin{array}{c}136 \\
(89,9 \%)\end{array}$ & $26(16,1 \%)$ & 162 & \multirow[t]{4}{*}{0,000} \\
\hline & 5 a 8 anos & $\begin{array}{c}324 \\
(95,3 \%)\end{array}$ & $\begin{array}{c}16 \\
(4,7 \%)\end{array}$ & 340 & & $\begin{array}{c}276 \\
(81,9 \%)\end{array}$ & $61(18,1 \%)$ & 337 & \\
\hline & 9 a 11 anos & $\begin{array}{c}407 \\
(98,6 \%)\end{array}$ & $6(1,4 \%)$ & 413 & & $\begin{array}{c}341 \\
(83,0 \%)\end{array}$ & $70(17,0 \%$ & 411 & \\
\hline & 12 anos ou mais & $\begin{array}{c}231 \\
(100 \%)\end{array}$ & $0(0 \%)$ & 231 & & $\begin{array}{c}223 \\
(96,5 \%)\end{array}$ & $8(3,5 \%)$ & 231 & \\
\hline \multirow{3}{*}{$\begin{array}{c}\text { Classe } \\
\text { socioeconômica }\end{array}$} & $A+B$ & $\begin{array}{c}262 \\
(100 \%)\end{array}$ & $0(0 \%)$ & 262 & 0,010 & $\begin{array}{c}251 \\
(96,2 \%)\end{array}$ & $10(3,8 \%)$ & 261 & \multirow[t]{3}{*}{0,000} \\
\hline & $\mathrm{C}$ & $\begin{array}{c}387 \\
(98,2 \%)\end{array}$ & $7(1,8 \%)$ & 394 & & $\begin{array}{c}338 \\
(86,4 \%)\end{array}$ & $53(13,6 \%)$ & 391 & \\
\hline & $\mathrm{D}+\mathrm{E}$ & $\begin{array}{c}447 \\
(95,7 \%)\end{array}$ & $\begin{array}{c}20 \\
(4,3 \%)\end{array}$ & 467 & & $\begin{array}{c}367 \\
(78,9 \%)\end{array}$ & $98(21,1 \%)$ & 465 & \\
\hline $\begin{array}{l}\text { Primeira } \\
\text { consulta }\end{array}$ & Sim & $\begin{array}{c}1072 \\
(98,1 \%)\end{array}$ & $\begin{array}{c}21 \\
(1,9 \%)\end{array}$ & 1093 & 0,038 & $\begin{array}{c}1072 \\
(86,0 \%)\end{array}$ & $174(14,0 \%)$ & 1246 & \multirow[t]{2}{*}{0,038} \\
\hline $\begin{array}{l}\text { odontológica/ } \\
\text { Posse de } \\
\text { escova dental } \\
\text { própria }\end{array}$ & Não & $\begin{array}{c}174 \\
(95,6 \%)\end{array}$ & $8(4,4 \%)$ & 182 & & $\begin{array}{c}21 \\
(72,4 \%)\end{array}$ & $8(27,6 \%)$ & 29 & \\
\hline
\end{tabular}

*Teste qui-quadrado

Tabela 3. Análise multivariada do desfecho posse de escova dental própria da amostra de 1282 crianças do Estudo SAÚDES, Vitória- ES, 2007 *

\begin{tabular}{|c|c|c|c|c|c|c|c|c|c|}
\hline \multirow{2}{*}{ Variáveis } & \multirow{2}{*}{ Categorias } & \multirow{2}{*}{$\begin{array}{l}\text { Coeficiente } \\
\text { beta }\end{array}$} & \multirow{2}{*}{$\begin{array}{c}\text { Erro } \\
\text { padrão }\end{array}$} & \multirow{2}{*}{$\begin{array}{c}\text { Teste } \\
\text { de Wald }\end{array}$} & \multirow{2}{*}{$\begin{array}{l}\text { Graus de } \\
\text { liberdade }\end{array}$} & \multirow{2}{*}{ p-valor } & \multirow{2}{*}{$\begin{array}{l}\text { Odds Ratio } \\
\text { ajustada }\end{array}$} & \multicolumn{2}{|c|}{$\begin{array}{c}\text { Intervalo de confiança } \\
95 \%\end{array}$} \\
\hline & & & & & & & & $\begin{array}{c}\text { Limite } \\
\text { Inferior }\end{array}$ & $\begin{array}{c}\text { Limite } \\
\text { Superior }\end{array}$ \\
\hline \multirow{4}{*}{ Idade } & 7 & & & & & & Referência & & \\
\hline & 8 & 1,195 & 0,545 & 4,807 & 1 & 0,028 & 3,303 & 1,135 & 9,610 \\
\hline & 9 & 1,239 & 0,550 & 5,083 & 1 & 0,024 & 3,452 & 1,176 & 10,137 \\
\hline & 10 & 1,932 & 0,790 & 5,986 & 1 & 0,014 & 6,901 & 1,468 & 32,434 \\
\hline Tabagismo & Sim & & & & & & Referência & & \\
\hline materno & Não & 1,506 & 0,409 & 13,527 & 1 & 0,000 & 4,509 & 2,021 & 10,060 \\
\hline Primeira & Não & & & & & & Referência & & \\
\hline $\begin{array}{c}\text { consulta } \\
\text { odontológica }\end{array}$ & Sim & 0,909 & 0,401 & 5,136 & 1 & 0,023 & 2,481 & 1,131 & 5,443 \\
\hline
\end{tabular}

*Modelo ajustado pelas variáveis: experiência de cárie, tipo de escola, raça/cor, aglomeração humana domiciliar, acesso a água filtrada e classe socioeconômica.

Tabela 4. Análise multivariada do desfecho primeira consulta odontológica da amostra de 1282 crianças do Estudo SAÚDES, Vitória- ES, $2007^{*}$

\begin{tabular}{|c|c|c|c|c|c|c|c|c|c|}
\hline \multirow[t]{2}{*}{ Variáveis } & \multirow[t]{2}{*}{ Categorias } & \multirow[t]{2}{*}{$\begin{array}{c}\text { Coeficiente } \\
\text { beta }\end{array}$} & \multirow[t]{2}{*}{$\begin{array}{c}\text { Erro } \\
\text { padrão }\end{array}$} & \multirow[t]{2}{*}{$\begin{array}{c}\text { Teste de } \\
\text { Wald }\end{array}$} & \multirow[t]{2}{*}{$\begin{array}{l}\text { Graus de } \\
\text { liberdade }\end{array}$} & \multirow[t]{2}{*}{ p-valor } & \multirow{2}{*}{$\begin{array}{c}\begin{array}{c}\text { Odds Ratio } \\
\text { ajustada }\end{array} \\
\text { Referência }\end{array}$} & \multicolumn{2}{|c|}{$\begin{array}{c}\text { Intervalo de } \\
\text { confiança } 95 \%\end{array}$} \\
\hline & & & & & & & & $\begin{array}{l}\text { Limite } \\
\text { Inferior }\end{array}$ & $\begin{array}{c}\text { Limite } \\
\text { Superior }\end{array}$ \\
\hline \multirow[t]{3}{*}{ Idade } & 8 & 0,334 & 0,257 & 1,695 & 1,000 & 0,193 & 1,396 & 0,845 & 2,309 \\
\hline & 9 & 0,842 & 0,280 & 9,015 & 1,000 & 0,003 & 2,321 & 1,340 & 4,022 \\
\hline & 10 & 1,149 & 0,319 & 12,950 & 1,000 & 0,000 & 3,155 & 1,687 & 5,899 \\
\hline
\end{tabular}


Tabela 4. Continuação...

\begin{tabular}{|c|c|c|c|c|c|c|c|c|c|}
\hline Variáveis & Categorias & $\begin{array}{c}\text { Coeficiente } \\
\text { beta }\end{array}$ & $\begin{array}{c}\text { Erro } \\
\text { padrão }\end{array}$ & $\begin{array}{c}\text { Teste de } \\
\text { Wald }\end{array}$ & $\begin{array}{r}\text { Graus de } \\
\text { liberdade }\end{array}$ & p-valor & $\begin{array}{c}\text { Odds Ratio } \\
\text { ajustada }\end{array}$ & \multicolumn{2}{|c|}{$\begin{array}{c}\text { Intervalo de } \\
\text { confiança } 95 \%\end{array}$} \\
\hline Tipo de & Pública & & & & & & Referência & & \\
\hline escola & Privada & 1,306 & 0,516 & 6,408 & 1,000 & 0,011 & 3,691 & 1,343 & 10,145 \\
\hline Quantidade & Um Quarto & & & & & & Referência & & \\
\hline de quartos & Dois ou mais & 0,552 & 0,250 & 4,861 & 1,000 & 0,027 & 1,736 & 1,063 & 2,835 \\
\hline Posse de & Não & & & & & & Referência & & \\
\hline $\begin{array}{c}\text { escova dental } \\
\text { própria }\end{array}$ & Sim & 0,725 & 0,365 & 3,935 & 1,000 & 0,047 & 2,065 & 1,009 & 4,226 \\
\hline
\end{tabular}

*Modelo ajustado pelas variáveis: experiência de cárie, raça/cor, tabagismo materno, residência conjunta pai e mãe, acesso a água filtrada e classe socioeconômica.

\section{DISCUSSÃO}

No presente estudo, foram captadas as variáveis relacionadas às características individuais infantis, maternas e socioeconômicas. Contudo, tais associações encontradas devem ser interpretadas com cautela, visto que a primeira consulta odontológica e a posse de escova dental própria foram as variáveis que mediram a dimensão do acesso realizado ${ }^{6}$, de maneira que as dimensões de qualidade e satisfação não foram objeto de estudo.

A utilização dos serviços odontológicos pela população brasileira varia conforme a região do país e condições socioeconômicas. Dados da Pesquisa Nacional de Saúde ${ }^{13}$ mostram que a proporção de indivíduos de 0 a 17 anos que realizaram consulta odontológica foi de $51,1 \%$. As Regiões Sul e Sudeste se destacaram com as maiores taxas $(55,8 \%$ e 53,0\%). Observou-se ainda que a proporção de pessoas que consultaram o dentista foi maior conforme o aumento do nível de escolaridade, maior rendimento domiciliar e residir em área urbana ${ }^{14}$.

No presente estudo, em relação às características individuais dos escolares, a idade associou-se com as duas variáveis dependentes utilizadas para determinar o acesso aos bens e serviços de saúde, visto que crianças na faixa etária de 9 a 10 anos possuíam, respectivamente, cerca de duas e três vezes mais chance de já terem realizado consulta odontológica em algum momento de suas vidas. De maneira similar, as chances de possuir escova dental própria aumentaram gradativamente nas idades de 8 (cerca de três vezes), 9 (cerca de 3,5 vezes) e 10 anos (cerca de sete vezes).

Esses achados corroboram outros estudos ${ }^{13,15,16}$, que evidenciam que as dificuldades no acesso à consulta odontológica diminuem com o aumento da idade, ou seja, crianças mais novas visitam o dentista com menos frequência do que as mais velhas. Um estudo realizado por Junqueira et al..$^{15}$ evidenciou que crianças com 5 anos de idade apresentam maiores dificuldades no acesso à consulta odontológica, quando comparadas a crianças de 12 e 15 anos. Aos 12 anos, mais de $30 \%$ das crianças apresentavam alguma dificuldade no acesso ao dentista. Já aos 15 anos, as dificuldades de acesso reduziram.

Crianças mais velhas e com problemas dentários possuem mais probabilidade de ir ao dentista do que aquelas sem problemas. As barreiras de acesso aos serviços de saúde bucal, a não inclusão das crianças em programas de educação e prevenção, podem levar a um retardo na procura por atendimento, ocasionando a busca pelo serviço apenas quando a condição se tornar dolorosa ${ }^{13}$. Assim, o fato de a criança possuir alguma enfermidade bucal pode propiciar também maior procura por algum tipo de cuidado bucal ${ }^{16}$.

Quanto aos aspectos sociais, o acesso aos serviços de saúde tende a refletir a iniquidade no uso de serviços odontológicos 7 . A relação do acesso aos serviços de saúde odontológicos com as características socioeconômicas vem sendo relatado por diferentes autores ${ }^{7,10}$. Neste estudo, o tipo de escola associou-se com a realização da primeira consulta odontológica, visto que os escolares matriculados em escolas privadas têm quase quatro vezes mais chance de já terem realizado consulta odontológica, quando comparados àqueles estudantes da rede pública de 
ensino. Achados similares foram encontrados por Oliveira et al. ${ }^{17}$, no qual alunos das escolas privadas procuraram 29\% vezes mais os serviços de saúde do que aqueles das escolas públicas, mostrando associações com melhores condições socioeconômicas.

Tal resultado é de extrema relevância, visto que escolares matriculados no ensino público podem representar um grupo de maior propensão à ocorrência de agravos bucais, devido a piores condições socioeconômicas ${ }^{18}$.

Deve-se considerar que o tipo de escola pode ser analisado como indicador socioeconômico, pois é necessário que a família da criança disponha de recursos financeiros para custear gastos diretos e indiretos relacionados ao ensino privado. Desse modo, a baixa renda é um fator estreitamente relacionado com o menor acesso aos serviços de saúde, podendo estar associada a poucos recursos financeiros ou até mesmo ao acesso à informação $0^{10}$ e a importância da manutenção da saúde bucal. Estudos ${ }^{10,13}$ mostram que a renda está relacionada à procura por consultas odontológicas. Indivíduos de melhores classes econômicas procuram o dentista mais vezes em relação aos grupos de indivíduos de menor renda e classe social.

Indivíduos com condição socioeconômica desfavorável tendem a procurar menos os serviços de saúde devido a barreiras de acesso relacionadas aos aspectos sociais e culturais. Além disso, a percepção da necessidade de tratamento e a importância atribuída à saúde influenciam a utilização de serviços pelos diferentes grupos sociais ${ }^{19}$.

No presente estudo, a classe socioeconômica foi utilizada no modelo final, sem interferência da escolaridade materna, mas não permaneceu estatisticamente significante. Há de se considerar que o tipo de escola que a criança frequenta pode não ser resultante apenas da classe socioeconômica na qual a criança foi categorizada. Nesta pesquisa, a classe socioeconômica foi determinada segundo níveis socioeconômicos da Associação Brasileira de Institutos de Pesquisa de Mercado (ABIPEME) ${ }^{20}$, que considera a posse de itens e a escolaridade do chefe da residência em sua classificação.

As classes determinadas pela ABIPEME talvez não tenham sido capazes de discriminar com precisão as diferenças entre os níveis socioeconômicos, medidos prioritariamente pela capacidade de consumo de bens. Por isso, o tipo de escola obteve maior capacidade de fazer essa distinção entre os níveis socioeconômicos.

Neste estudo, crianças com residências que possuíam dois ou mais quartos também apresentavam quase duas vezes mais chance de terem realizado a primeira consulta odontológica em relação àqueles com residência de apenas um quarto. Resultados semelhantes foram encontrados no estudo de Noro et al. ${ }^{21}$, em que crianças com melhores condições de moradia tiveram também mais chance de terem utilizado o serviço de saúde odontológico.

A condição econômica é um fator intimamente relacionado com o menor acesso aos serviços, podendo estar combinado a pouco acesso à informação de saúde desta população ${ }^{10}$. 0 fato de as crianças residirem em casas maiores pode ser indicativo de exposição a uma situação socioeconômica mais favorável da família em algum momento da vida. Melhores condições socioeconômicas podem propiciar um maior acesso às informações a respeito da saúde bucal 22 .

Considerando o contexto familiar, o comportamento em saúde bucal dos familiares, em especial o materno, pode influenciar na adoção de hábitos benéficos à saúde e no cuidado e engajamento na saúde oral por parte das crianças $^{22}$. Neste estudo, o tabagismo materno demonstrou associação significativa com posse de escova dental própria. No grupo de crianças em que a mãe não fumava, a chance de ter escova dental própria foi quase cinco vezes maior em relação àqueles menores em que a mãe era tabagista.

O consumo do tabaco pode coexistir com outros comportamentos de risco ${ }^{23}$. 0 fato de a mãe não fumar pode estar relacionado a práticas e hábitos de vida mais saudáveis adotados pela família, resultando em uma maior preocupação também com a correta higienização da cavidade bucal. 
Também se deve destacar que a posse de escova dental própria e a primeira consulta odontológica foram variáveis altamente associadas entre si. 0 fato de ter escova dental própria aumentou a chance de as crianças terem ido ao dentista, assim como ter ido ao dentista aumentou a chance de os escolares terem escova dental própria

Os hábitos de cuidado com a saúde, da mesma maneira que os fatores de risco, geralmente estão relacionados entre $\mathrm{si}^{24}$. Ter ido ao dentista em algum momento da vida pode vir a contribuir positivamente para a incorporação de hábitos de higiene bucal mais adequados, e a posse de escova dental própria é essencial para que a higiene bucal ocorra de maneira correta. De modo similar, aquelas crianças que possuem escova própria também podem estar mais propensas a escovarem os dentes e a estenderem esse cuidado sob a forma de visitas regulares ao dentista.

0 presente estudo apresenta limitações inerentes ao momento da coleta dos dados, de maneira que algumas informações podem estar sujeitas a viés de informação e memória tanto por parte das crianças quanto por parte dos responsáveis pelos escolares. Além disso, as associações encontradas devem ser analisadas com cautela, visto que o estudo não pretende realizar inferências diretas entre causas e efeitos, por se tratar de um estudo transversal. Possui como vantagem o fato de que, ao considerar em seu delineamento os escolares, conseguiu abranger quase a totalidade de crianças de 7 a 10 anos, visto que $97,53 \%{ }^{25}$ dos menores dessa faixa etária estão matriculados no sistema de ensino público e privado.

\section{CONCLUSÃO}

Os resultados deste estudo apontam para a desigualdade no acesso às ações e serviços de saúde odontológicos e chamam atenção para a necessidade de novos estudos relacionados à acessibilidade à saúde bucal. Crianças mais velhas, filhas de mães não fumantes e que já foram ao dentista alguma vez na vida, apresentam maior acesso a escova dental própria como bem de saúde. Além disso, o fato de a criança ser estudante de escola privada, ser mais velha, ter residência maior e ter escova dental própria, também contribui para o acesso à primeira consulta odontológica.

A equipe interdisciplinar apresenta papel fundamental nesse contexto de promoção da saúde bucal das crianças. Os achados sugerem a necessidade de melhorias na identificação das crianças e captação precoce para os serviços de saúde bucal.

Sabe-se também que o acesso aos bens e serviços de saúde tende a refletir os níveis de desigualdades existentes na sociedade, e que cada grupo social apresenta necessidades de cuidado em saúde distintas. Neste sentido, as políticas públicas de saúde bucal coletiva devem ser capazes de eliminar as barreiras relacionadas ao acesso aos serviços de saúde, tornando o acesso a bens e serviços de saúde mais igualitários, a fim de garantir a saúde como direito social, por meio da elaboração de estratégias compensatórias, visando diminuir as desigualdades e os riscos a que as classes menos favorecidas estão expostas.

\section{REFERÊNCIAS}

1. Giovanella L, Escorel S, Lobato LVC, Noronha JC, Carvalho AI, editores. Políticas e sistema de saúde no Brasil. Rio de Janeiro: Fiocruz; 2012.

2. Penchansky R, Thomas JW. The concept of access: definition and relationship to consumer satisfaction. Med Care. 1981 Feb;19(2):127-40. http://dx.doi.org/10.1097/00005650-198102000-00001. PMid:7206846.

3. Travassos C, Martins M. Uma revisão sobre os conceitos de acesso e utilização de serviços de saúde. Cad Saude Publica. 2004;20(Supl. 2):S190-8. http://dx.doi.org/10.1590/S0102-311X2004000800014. PMid:15608933. 
4. Donabedian A. Aspects of medical care administration: Specifying Requirements for Health Care. Cambridge: Harvard University Press; 1973.

5. Andersen R, Newman JF. Societal and individual determinants of medical care utilization in the United States. Milbank Mem Fund Q Health Soc. 1973;51(1):95-124. http://dx.doi.org/10.2307/3349613. PMid:4198894.

6. Andersen RM. Revisiting the behavioral model and access to medical care: does it matter? J Health Soc Behav. 1995 Mar;36(1):1-10. http://dx.doi.org/10.2307/2137284. PMid:7738325.

7. Palma ABO, Ferreira RC, Martins AMEB, Assis KBO, Duarte DA. Determinantes do não uso de serviços odontológicos por crianças de cinco anos. Arq Odontol. 2015 Jan-Mar;51(1):14-24.

8. Mclntyre D, Mooney G, editores. Aspectos econômicos da equidade em saúde. Rio de Janeiro: Fiocruz; 2014.

9. Louvison MCP, Lebrão ML, Duarte YAO, Santos JLF, Malik AM, Almeida ES. Desigualdades no uso e acesso aos serviços de saúde entre idosos do município de São Paulo. Rev Saude Publica. 2008 Ago;42(4):733-40. http://dx.doi.org/10.1590/S0034-89102008000400021. PMid:18709246.

10. Comassetto MO, Baumgarten A, Kindlein KA, Hilgert JB, Figueiredo MC, Faustino-Silva DD. Acesso à saúde bucal na primeira infância no município de Porto Alegre, Brasil. Cien Saude Colet. 2019 Mar;24(3):953-61. http://dx.doi.org/10.1590/1413-81232018243.29082016. PMid:30892516.

11. Vitória. Prefeitura Municipal. Serviços odontológicos [Internet]. Vitória: Prefeitura Municipal de Vitória; 2017 [citado em 2021 Jun 7]. Disponível em: https://m.vitoria.es.gov.br/cidadao/servicosodontologicos

12. Faria CP, Cade NV, Nascimento LR, Molina MCB. Prevalência de excesso de peso em crianças de sete a dez anos de Vitória-ES, Brasil: um estudo de base escolar. Revista Brasileira de Pesquisa em Saúde. 2011;13(1):31-7.

13. Barasuol JC, Garcia LP, Freitas RC, Dalpian DM, Menezes JVNB, Santos BZ. Dental care utilization among children in Brazil: an exploratory study based on data from national household surveys. Cien Saude Colet. 2019 Feb;24(2):649-57. http://dx.doi.org/10.1590/1413-81232018242.03232017. PMid:30726397.

14. Instituto Brasileiro de Geografia e Estatística - IBGE. Pesquisa Nacional de Saúde 2019 [Internet]. Rio de Janeiro: IBGE; 2019 [citado em 2021 Jun 7]. Disponível em: https://biblioteca.ibge.gov.br/visualizacao/livros/liv101748.pdf

15. Junqueira SR, Frias AC, Zilbovicius C, Araujo ME. Saúde bucal e uso dos serviços odontológicos em função do Índice de Necessidades em Saúde: São Paulo, 2008. Cien Saude Colet. 2012 Abr;17(4):101524. http://dx.doi.org/10.1590/S1413-81232012000400023. PMid:22534855.

16. Finlayson TL, Chuang E, Baek J-D, Seidman R. Dental service utilization among children in the child welfare system. Matern Child Health J. 2018 May;22(5):753-61. http://dx.doi.org/10.1007/s10995018-2444-y. PMid:29423585.

17. Oliveira MM, Andrade SSCA, Campos MO, Malta DC. Fatores associados à procura de serviços de saúde entre escolares brasileiros: uma análise da Pesquisa Nacional de Saúde do Escolar (PeNSE), 2012. Cad Saude Publica. 2015 Ago;31(8):1603-14. http://dx.doi.org/10.1590/0102-311X00165214. PMid:26375640.

18. Oliveira RCN, Souza JGS, Oliveira CC, Oliveira LFB, Popoff DAV, Martins AMEBL, et al. Acesso a orientações de higiene bucal entre escolares da rede pública de ensino. Rev Odontol UNESP. 2014 NovDez;43(6):414-20. http://dx.doi.org/10.1590/1807-2577.1042.

19. Rohr RIT, Barcellos LA. As barreiras de acesso para os serviços odontológicos. UFES Rev Odontol. 2008;3(10):37-41.

20. Associação Brasileira dos Institutos de Pesquisa de Mercado - ABIPEME. Critério Padrão de Classificação Econômica Brasil - 2008. São Paulo: ABIPEME. 
21. Noro LRA, Roncalli AG, Mendes FIR Jr, Lima KC. A utilização de serviços odontológicos entre crianças e fatores associados em Sobral, Ceará, Brasil. Cad Saude Publica. 2008 Jul;24(7):1509-16. http://dx.doi.org/10.1590/S0102-311X2008000700005. PMid:18670674.

22. Castilho ARF, Mialhe FL, Barbosa TS, Puppin-Rontani RM. Influência do ambiente familiar sobre a saúde bucal de crianças: uma revisão sistemática. J Pediatr. 2013 Abr;89(2):116-23. http://dx.doi.org/10.1016/j.jped.2013.03.014. PMid:23642420.

23. Barreto SM, Giatti L, Casado L, Moura L, Crespo C, Malta DC. Exposição ao tabagismo entre escolares no Brasil. Cien Saude Colet. 2010 Out;15(Supl. 2):3027-34. http://dx.doi.org/10.1590/S141381232010000800007. PMid:21049143.

24. Davoglio RS, Aerts DRG, Abegg C, Freddo SL, Monteiro L. Fatores associados a hábitos de saúde bucal e utilização de serviços odontológicos entre adolescentes. Cad Saude Publica. 2009 Mar;25(3):655-67. http://dx.doi.org/10.1590/S0102-311X2009000300020. PMid:19300854.

25. Paredes SO, Almeida DB, Fernandes JMFA, Forte FDS, Sampaio FC. Behavioral and social factors related to dental caries in 3 to 13 year-old children from João Pessoa, Paraíba, Brazil. Rev Odonto Ciênc. $2009 ; 24(3): 231-5$.

\section{CONFLITOS DE INTERESSE}

Os autores declaram não haver conflitos de interesse.

\section{*AUTOR PARA CORRESPONDÊNCIA}

Edson Theodoro dos Santos Neto, UFES - Universidade Federal do Espírito Santo, Centro de Ciências da Saúde, Programa de Pós-graduação em Saúde Coletiva, Av. Marechal Campos, 1468, Maruípe, 29040-090 Vitória - ES, Brasil, e-mail: edsontheodoroneto@uol.com.br

Recebido: Junho 23, 2021

Aprovado: Agosto 12, 2021 